\title{
Zeolite Improves High-Fat Diet-Induced Hyperglycemia, Hyperlipidemia and Obesity in Mice
}

\author{
Kazuhiro KuBO $^{1}$ and Yasuyuki KAWAI ${ }^{2}$ \\ ${ }^{1}$ Department of Home Economics, Faculty of Education, Gifu University, Yanagido, Gifu 501-1193, Japan \\ ${ }^{2}$ Bikensangyo Co., Ltd., Kanare, Meitou-ku, Nagoya, Aichi 465-0005, Japan
}

(Received November 23, 2020)

\begin{abstract}
Summary Zeolite, an abundant mineral in the Earth's crust, is utilized in a wide range of fields because of its well-known adsorption properties. Its application as a functional food ingredient resembling dietary fiber is expected, but it has not yet been investigated in the context of prevention of lifestyle-related diseases. The present study was designed to evaluate the availability and safety of a natural zeolite preparation for this purpose. Acute oral toxicity testing showed that the lowest lethal dose of zeolite was more than 2,000 mg/kg body weight for both male and female mice. In a prolonged feeding test for $18 \mathrm{wk}$ using model mice with high-fat-induced obesity and type 2 diabetes mellitus, intake of a $10 \%$ zeolite-containing diet suppressed body weight gain, as well as liver and visceral fat weights, without any changes in food and energy intake. Moreover, plasma lipid (triacylglycerol, total cholesterol and high-density-lipoprotein cholesterol) levels and fasting blood glucose levels decreased in parallel with zeolite intake. No changes in the glycated hemoglobin level were found. However, in an oral glucose tolerance test at week 12, increased postprandial blood glucose levels were suppressed in accordance with zeolite intake, and then insulin secretion was also decreased. On the other hand, a decrease of plasma amylase activity and increases in total bilirubin and urea nitrogen suggested the need for further investigation of safety.
\end{abstract}

Key Words zeolite, LDLo, hyperglycemia, hyperlipidemia, insulin secretion, postprandial blood glucose, type 2 diabetes mellitus, obesity

Natural zeolites-abundant minerals in the Earth's crust-are aluminosilicates possessing ion-exchange properties due to their fine network of structural cavities linked through common oxygen atoms (1). As they selectively adsorb water and exchange cations $(2,3)$, zeolites have been widely applied as adsorbents, ion exchangers and catalysts in industry, agriculture, veterinary medicine, sanitation and environmental protection. In addition, zeolites can reduce the level of blood glucose as a result of their well-known adsorption properties (4-7) and have the ability to absorb both lipids (8) and proteins. In the context of type 2 diabetes mellitus, functional properties such as modification of intestinal absorption due to dietary fiber (9) are of interest. In fact, in rats injected with streptozocin, the main ingredient of zeolite prevents the development of diabetes and protects pancreatic beta cells from damage (10). The zeolite is not absorbed through the gastrointestinal tract (1). Therefore, zeolite appears to be applicable as a functional food material like dietary fiber and indigestible dextrin if its efficacy and safety can be confirmed. The natural zeolites characterized so far have considerably low cost and exhibit no toxicity and cause no environmental pollution. The mineral assemblies of the most common zeolite occurrences in nature are clinoptilolite- and mordenite containing tuffs, in which the

E-mail: kubochan@gifu-u.ac.jp clinoptilolite and mordenite content is high $(80 \%$ and over) (11). Clinoptilolite, mordenite and non-fibrous Japanese zeolite are classified as Group 3 (not classifiable as carcinogenic to humans) by the International Agency for Research on Cancer (IARC) Monographs, Volumes 1-129. (Available online: https://monographs. iarc.who.int/list-of-classifications (accessed on 2 August 2021).) European Food Safety Authority (EFSA) concluded that 10,000 mg clinoptilolite/kg complete feed could be considered safe for all animal species (12). Oral consumption of clinoptilolite, due to its extreme chemical stability, does not represent a potential risk for in vivo applications (13). However, the applications and benefits of zeolite as a food material should be investigated carefully.

The present study was designed to evaluate 1) the toxicity of a new natural form of zeolite and 2) its availability for prevention of high-fat diet-induced obesity and type 2 diabetes mellitus in mice.

\section{MATERIALS AND METHODS}

Zeolite. The natural zeolite used in this study was from igneous rock found in the Yatsuo area, Toyama prefecture, Hokuriku province, central Japan. After being passed through a $150-\mu \mathrm{m}$ mesh sieve, the dry powder with a mean particle diameter of $104 \mu \mathrm{m}$ was provided for this study by Bikensangyo Co., Ltd. (Nagoya, Japan). The mineral contents of $100 \mathrm{~g}$ of dry 
zeolite powder, and the methods used to measure them, were as follows. Silicon $24.5 \mathrm{~g}$ (gravimetry), potassium $2.54 \mathrm{~g}$ and sodium $2.08 \mathrm{~g}$ (atomic absorption spectrophotometry (AAS)), iron $930 \mathrm{mg}$, calcium $829 \mathrm{mg}$, magnesium $321 \mathrm{mg}$, manganese $25.7 \mathrm{mg}$, phosphorus $5.1 \mathrm{mg}$, zinc $2.97 \mathrm{mg}$, copper $0.29 \mathrm{mg}$, total chromium not-detectable (nd), and vanadium nd (inductively coupled plasma atomic emission spectroscopy (ICP-AES)), selenium nd (fluorometry), and iodine nd (gas chromatography).

Acute oral toxicity test.

Ethical approval. The study (Issuance No.: 309080017-002) was approved and performed at Japan Food Research Laboratories (Tokyo, Japan) according to the protocols stipulated in OECD Guideline 420, 2001 (14).

Preliminary feeding. Five-week-old ICR male and female mice were purchased from Japan SLC, Inc. (Tokyo, Japan) and maintained under standard conditions of $23 \pm 2^{\circ} \mathrm{C}$ and $55 \pm 15 \%$ humidity with a $12 \mathrm{~h} /$ $12 \mathrm{~h}$ light/dark cycle. Each polycarbonate cage housed five mice, which had ad libitum access to tap water and a basal diet (Labo MR Stock; Nosan Corporation LifeTech Department, Yokohama, Japan) during the acclimation period. After the general condition of the animals had been confirmed to be normal after $7 \mathrm{~d}$ of acclimation, the mice were used for the study.

Administration. The zeolite powder was suspended in water for injection, and test solutions of $100 \mathrm{mg} / \mathrm{mL}$ were prepared. Before administration, the mice were fasted for $4 \mathrm{~h}$. After measurement of body weight, the study group $(n=5)$ were forcibly administered a single oral dose of $2,000 \mathrm{mg} / 20 \mathrm{~mL} / \mathrm{kg}$ body weight (bw) using a gastric tube according to the dosage stipulated in OECD Guideline 420, 2001 (14). The control group $(n=5)$ were given water for injection at $20 \mathrm{~mL} / \mathrm{kg}$ bw using a similar procedure.

Monitoring. During a monitoring period of $14 \mathrm{~d}$, the mice were observed frequently on the first day and then observed once per day from the second day onwards. Body weight was measured on the 7 th and 14 th days. After the monitoring period, all the animals were euthanatized and necropsied.

Prolonged feeding test.

Ethical approval. The study's protocol was approved by the Animal Care and Use Committee of Gifu University (Approval No.: 29-103). All measures were taken to minimize the animals' pain and suffering.

Feeding conditions. The mice were maintained under standard conditions of $23 \pm 2{ }^{\circ} \mathrm{C}$ and $55 \pm 15 \%$ humidity with a 12 h/12 h light/dark cycle. Each cage housed seven mice. Following a 7 -d acclimation period on the basal diet, the mice were maintained ad libitum on the experimental diets and tap water for $18 \mathrm{wk}$ (126 d).

Animals and diets. Five-week-old male C57BL/ 6JJmsSlc mice $(n=28)$ were purchased from Japan SLC, Inc. (Shizuoka, Japan). The mice had ad libitum access to tap water and a basal diet (Rodent Diet CE-2; CLEA Japan, Inc.) during the 7-d acclimation period. Experi- mental diets were based on a high-fat diet for studies of diabetes and obesity, Quick Fat (CLEA Japan, Inc.) [24 kcal\% protein, $46 \mathrm{kcal} \%$ carbohydrate, $30 \mathrm{kcal} \%$ fat (fat source: beef tallow and an embryo)], which was uniformly mixed with the zeolite powder at ratios of $0 \%$ (control), $0.1 \%, 1 \%$, and $10 \%$. The combination ratio of the zeolite in foods was set according to OECD Guideline 408, 2018 (15). A limit test may be performed if no effects are expected at a dose of $1,000 \mathrm{mg} / \mathrm{kg} \mathrm{bw} / \mathrm{d}$ (15). One thousand milligrams zeolite/kg bw/d corresponds to the Ze 1\% group in this study. For chemicals administered via the diet it is important to ensure that the palatability of the test chemical involved does not interfere with normal nutritional balance (15). Therefore, four groups were set at $10 \%$ of maximal doses, with a dose width of 10 times. A level of $10 \%$ is equal to a safe intake of the zeolite clinoptilolite for all animal species, as determined by the EFSA (12). All of the diets were pelleted. All of the following tests were performed with seven mice per group.

Fasting blood glucose. The fasting blood glucose level was measured on the 1st day, and at 4 th, 8th, 12 th, 16th, and 18th weeks. After an overnight fast, the blood glucose level was measured using a blood glucose monitor (FreeStyle Freedom Lite; Nipro Co., Osaka, Japan).

OGTTs. Oral glucose tolerance tests (OGTTs) were performed at the 12th and 16th weeks. Following an overnight fast, the time-course of the glucose level in tail vein blood was measured using the FreeStyle Freedom Lite (Nipro Co.) 0, 30, 60, 120, and 180 min after oral administration of $20 \%$ glucose solution $(2 \mathrm{~g} / \mathrm{kg}$ bw). Changes in the postprandial blood glucose level in the OGTTs were measured as the total area under the curve (tAUC) and the incremental area under the curve (iAUC). For measurements of insulin secretion, blood from the tail vein was collected into capillary tubes containing lithium-heparin. The blood samples were then centrifuged $(15,000 \times g$ for $15 \mathrm{~min})$ to separate the plasma, and the plasma insulin level was measured using a Mouse Insulin ELISA Kit (Morinaga Institute of Biological Science, Inc., Kanagawa, Japan).

ITTs. The insulin tolerance tests (ITTs) were performed to assess insulin sensitivity at the 14th week. Following an overnight fast, mice received an intraperitoneal injection of human insulin $(1 \mathrm{U} / \mathrm{kg}$; Eli Lilly Japan K.K., Japan). The blood glucose levels were measured using the FreeStyle Freedom Lite (Nipro Co.) after 0, 30, 60, 120, and $180 \mathrm{~min}$.

Treatment of tissues. After $18 \mathrm{wk}$, the 24-wk-old mice were sacrificed by cardiac puncture under deep anesthesia (100 mg/kg sodium pentobarbital intraperitoneally) after an overnight fast. The liver, kidneys, pancreas, perirenal fat, and epididymal fat were resected and weighed immediately. A portion of the total blood was used for determination of the glycated hemoglobin (HbA1c) concentration. The plasma was fractionated from a portion of the blood by centrifugal separation and used for determination of other biochemical parameters. 
Biochemical parameters. HbA1c concentration was determined with a Quo-Lab HbAlc Analyzer (Nipro Co.). A Hitachi 7180 automatic analyzer (Hitachi High-Technologies Co., Japan) and testing kits (FUJIFILM Wako Pure Chemical Corporation, Osaka, Japan) were used to determine the levels of amylase (L-type amylase), alanine aminotransferase (L-type ALT·J2), aspartate aminotransferase (L-type AST·J2), albumin (Albumin-II HA Test Wako), total protein (Total Protein-HR2), total cholesterol (L-type $\mathrm{CHO} \cdot \mathrm{H})$, creatinine (L-type CRE·M), lactate dehydrogenase (L-type LD·J), non-esterified fatty acids (NEFA-HR), and triacylglycerols (L-type TG·M). Sodium, potassium, and chloride were determined with reagents for a Hitachi ion-selective electrode using the ion-selective electrode method. Inorganic phosphorus levels were measured using an assay kit (IP-II; Kyowa MEDEX Co., Ltd., Tokyo, Japan). Total bilirubin levels were measured with Nescoat VL T-BIL (Alfresa Pharma Co., Osaka, Japan). Blood urea nitrogen was measured with a UN-S kit employing a urease and glutamate dehydrogenase assay (Denka Seiken, Tokyo, Japan). Calcium levels were measured using a calcium assay kit, Accuras Auto Ca, which is based on $p$-nitrophenol enzymatically released by phospholipase D (Shino-test Co., Ltd., Sagamihara, Japan). Concentrations of high-density lipoprotein cholesterol (HDL-c; Cholestest ${ }^{\circledR}$ N HDL; Sekisui Medical Co., Ltd., Japan) and low-density lipoprotein cholesterol (LDL-c; Cholestest ${ }^{\circledR}$ LDL; Sekisui Medical Co., Ltd.) were determined using the direct method. The LDL-c/HDL-c ratio was calculated as a marker of dyslipidemia. In addition, rectal temperature was measured regularly.

Statistical analysis. Differences between two groups in the acute oral toxicity test were compared by un- paired $t$-test at a significance level of $<5 \%$. In the prolonged feeding test, comparison of differences between the zeolite intake groups and the control group was conducted using a post hoc Dunnett's multiple-comparisons test following one-way ANOVA. The BellCurve for Excel (version 3.2) statistical software package was used for all statistical analyses.

\section{RESULTS}

Acute oral toxicity test

No abnormality or death was observed in any of the male and female mice utilized for this study. There were no significant differences in body weight between the zeolite intake groups and the control group during the monitoring period (Table 1 ). With regard to color of feces and urine, no differences between the groups were observed.

\section{Prolonged feeding test}

Toxicity and rectal temperature. None of the animals utilized for this study became ill or died prior to the

Table 1. Changes in the body weight $(\mathrm{g})$ of male and female mice after oral administration of zeolite solution or water (control).

\begin{tabular}{cccc}
\hline Group & Before & After 7 d & After 14d \\
\hline Male & & & \\
$\quad$ Control & $32.2 \pm 1.0$ & $36.2 \pm 1.4$ & $38.6 \pm 1.7$ \\
Zeolite & $32.6 \pm 1.1$ & $36.7 \pm 1.3$ & $39.5 \pm 1.4$ \\
Female & & & \\
Control & $25.0 \pm 0.5$ & $28.4 \pm 0.8$ & $31.7 \pm 0.9$ \\
Zeolite & $25.1 \pm 0.7$ & $27.9 \pm 1.2$ & $30.8 \pm 2.0$ \\
\hline
\end{tabular}

The values are presented as mean $\pm \operatorname{SE}(n=5)$.

Table 2. Food intake, energy intake, water intake, body weight and tissue weights in mice with high-fat diet-induced obesity.

\begin{tabular}{|c|c|c|c|c|}
\hline Parameters & Ze $0 \%$ & Ze $0.1 \%$ & Ze $1 \%$ & Ze $10 \%$ \\
\hline Food intake $(\mathrm{g} / \mathrm{d})$ & $3.96 \pm 0.06$ & $3.85 \pm 0.06$ & $4.15 \pm 0.09$ & $4.15 \pm 0.11$ \\
\hline Total food intake (g/18 wk) & $498.61 \pm 9.70$ & $485.42 \pm 8.89$ & $523.21 \pm 12.63$ & $523.23 \pm 21.80$ \\
\hline Energy intake (kcal/d) & $16.2 \pm 0.3$ & $15.7 \pm 0.3$ & $16.8 \pm 0.4$ & $15.3 \pm 0.6$ \\
\hline Total energy intake (kcal/18 wk) & $2,040.8 \pm 39.7$ & $1,983.9 \pm 36.4$ & $2,119.1 \pm 51.2$ & $1,926.5 \pm 80.3$ \\
\hline Water intake $(\mathrm{g} / \mathrm{d})$ & $5.10 \pm 0.22$ & $5.06 \pm 0.26$ & $5.36 \pm 0.26$ & $5.85 \pm 0.28$ \\
\hline Total water intake (g/18 wk) & $642.45 \pm 28.02$ & $637.52 \pm 33.10$ & $674.79 \pm 32.76$ & $736.81 \pm 35.05$ \\
\hline Initial body weight (g) & $23.23 \pm 0.20$ & $23.20 \pm 0.25$ & $22.94 \pm 0.31$ & $23.21 \pm 0.33$ \\
\hline Final body weight $(\mathrm{g})$ & $44.84 \pm 1.34$ & $44.19 \pm 1.01$ & $43.19 \pm 1.93$ & $39.36 \pm 1.13^{*}$ \\
\hline Body weight gain (g/18 wk) & $21.61 \pm 1.30$ & $20.99 \pm 1.19$ & $20.24 \pm 2.06$ & $16.14 \pm 1.38^{*}$ \\
\hline Liver $(\mathrm{g})$ & $2.19 \pm 0.34$ & $2.04 \pm 0.15$ & $1.98 \pm 0.20$ & $1.49 \pm 0.06^{*}$ \\
\hline Liver (g/100 g bw) & $4.79 \pm 0.56$ & $4.59 \pm 0.25$ & $4.52 \pm 0.26$ & $3.78 \pm 0.08$ \\
\hline Kidneys (g) & $0.45 \pm 0.02$ & $0.44 \pm 0.02$ & $0.50 \pm 0.02^{*}$ & $0.48 \pm 0.01$ \\
\hline Kidneys (g/100 g bw) & $1.01 \pm 0.02$ & $1.01 \pm 0.03$ & $1.18 \pm 0.09^{*}$ & $1.23 \pm 0.06^{*}$ \\
\hline Pancreas $(\mathrm{g})$ & $0.28 \pm 0.02$ & $0.31 \pm 0.02$ & $0.34 \pm 0.03$ & $0.28 \pm 0.01$ \\
\hline Pancreas (g/100 g bw) & $0.62 \pm 0.04$ & $0.70 \pm 0.04$ & $0.78 \pm 0.06^{*}$ & $0.72 \pm 0.03$ \\
\hline Epididymal fat $(\mathrm{g})$ & $2.56 \pm 0.05$ & $2.46 \pm 0.08$ & $2.16 \pm 0.26$ & $1.79 \pm 0.22^{* *}$ \\
\hline Epididymal fat (g/100 g bw) & $5.74 \pm 0.15$ & $5.61 \pm 0.26$ & $4.90 \pm 0.43$ & $4.48 \pm 0.47^{*}$ \\
\hline Perirenal fat $(\mathrm{g})$ & $0.84 \pm 0.06$ & $0.80 \pm 0.04$ & $0.74 \pm 0.11$ & $0.61 \pm 0.08$ \\
\hline Perirenal fat (g/100 g bw) & $1.88 \pm 0.11$ & $1.81 \pm 0.05$ & $1.67 \pm 0.20$ & $1.53 \pm 0.17$ \\
\hline
\end{tabular}


experimental endpoint. No inter-group differences in murine rectal temperature or its daily variance were observed (data not shown). Upon autopsy, no megacolon or macroscopic abnormalities were observed.

Waste, food, energy and water intake, and body and tissue weight. All animals in the zeolite intake groups accepted and tolerated the zeolite-supplemented feed without problems. The color of the feces in the control group was dark brown, but that of the Ze $10 \%$ group was light gray. With regard to urine color, no differences between the groups were observed. No constipation was noted. Food intake, energy intake, water intake, body weight, and tissue weights are shown in Table 2 and Fig. S1 (Supplemental Online Material). There were no significant inter-group differences in initial body weight, or food, energy or water intake. In contrast, final body weight and body weight gain decreased in parallel with increased zeolite intake, the decrease being significant in the $10 \%$ zeolite intake group (Ze 10\%) relative to the Ze $0 \%$ group. There were no significant inter-group differences in total food intake (Fig. 1) and total water intake (Fig. S1) at each week. The initial significant difference (inflection point) in body weights between the Ze $0 \%$ and Ze 10\% groups was observed in the 10th

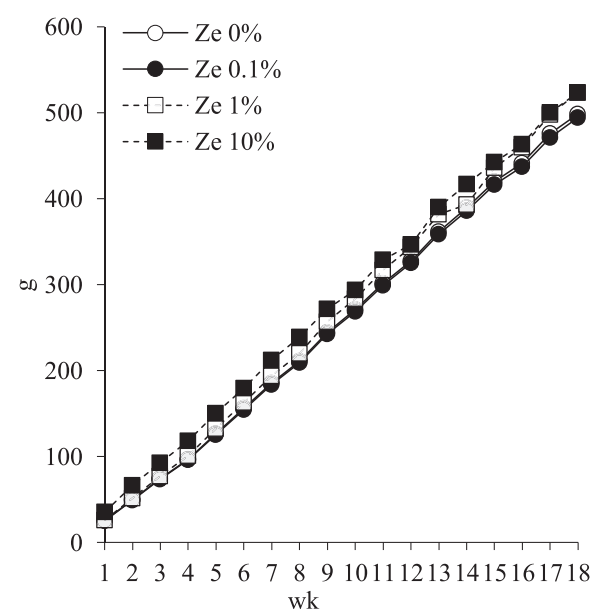

Fig. 1. Changes in total food intake in mice with highfat diet-induced obesity. The values are presented as the mean for each week $(n=7)$. week (Fig. 2). Hardly any changes in pancreas and kidney weight were observed. On the other hand, the weights of the liver, epididymal fat and perirenal fat decreased in parallel with increased zeolite intake, the decrease in the former two being significant in the Ze $10 \%$ group relative to the Ze $0 \%$ group.

Fasting blood glucose and HbA1c. Changes in the fasting blood glucose level and HbA1c level are shown in Table 3. The fasting blood glucose level was significantly decreased in the Ze 10\% group relative to the Ze $0 \%$ group after 4, 12 and $16 \mathrm{wk}$. However, no significant difference was evident after 8 and $18 \mathrm{wk}$. There was also no significant inter-group difference in $\mathrm{HbA} 1 \mathrm{c}$ level at the 18th week.

Postprandial blood glucose, insulin secretion and sensitivity. In the OGTTs at the 12th week, the postprandial blood glucose levels in the Ze 10\% group were significantly lowered relative to those in the Ze $0 \%$ group at all measurement points (Fig. 3). Both tAUC and iAUC reflected these changes and were significantly decreased in the Ze $10 \%$ group relative to the Ze 0\% group (Table 4). The plasma insulin levels in the Ze 10\% group were significantly reduced relative to the Ze $0 \%$ group at all measurement points (Fig. 4). On the other hand, ITTs at

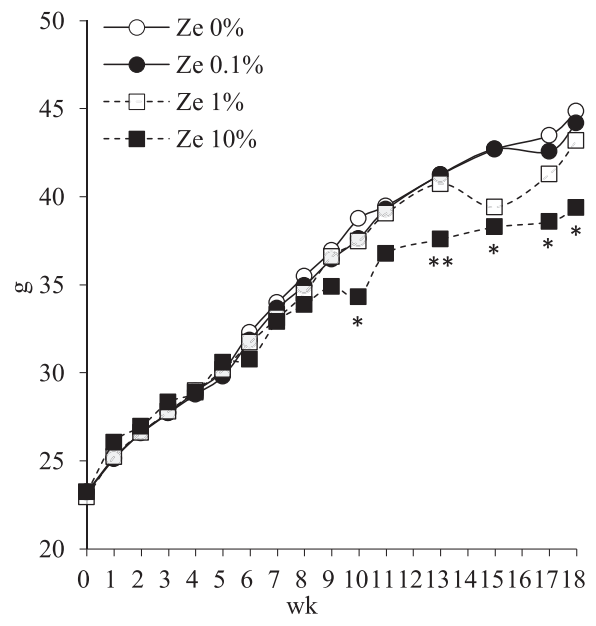

Fig. 2. Changes in body weight in mice with high-fat diet-induced obesity. The values are presented as the mean for each week $(n=7)$. $p$-value vs. Ze 0\% (Dunnett's test). ${ }^{*} p<0.5,{ }^{* *} p<0.01$.

Table 3. Changes in fasting blood glucose and hemoglobin A1c levels in mice with high-fat diet-induced obesity.

\begin{tabular}{|c|c|c|c|c|c|}
\hline Parameters & & Ze $0 \%$ & Ze $0.1 \%$ & Ze $1 \%$ & Ze $10 \%$ \\
\hline \multirow[t]{6}{*}{ Fasting blood glucose $(\mathrm{mmol} / \mathrm{L})$} & week 0 & $10.4 \pm 0.4$ & $10.2 \pm 0.4$ & $10.1 \pm 0.3$ & $10.1 \pm 0.3$ \\
\hline & week 4 & $11.1 \pm 0.4$ & $12.5 \pm 0.4$ & $10.3 \pm 0.6$ & $9.31 \pm 0.6^{*}$ \\
\hline & week 8 & $12.3 \pm 0.7$ & $13.1 \pm 0.6$ & $11.8 \pm 0.6$ & $11.4 \pm 0.6$ \\
\hline & week 12 & $8.3 \pm 1.1$ & $7.3 \pm 0.3$ & $7.3 \pm 0.3$ & $5.2 \pm 0.3^{* *}$ \\
\hline & week 16 & $11.5 \pm 0.4$ & $10.4 \pm 0.5$ & $10.3 \pm 0.4$ & $8.5 \pm 0.8^{* *}$ \\
\hline & week 18 & $10.9 \pm 0.6$ & $11.4 \pm 0.3$ & $9.9 \pm 0.7$ & $10.9 \pm 0.6$ \\
\hline \multirow{2}{*}{\multicolumn{2}{|c|}{$\begin{array}{l}\text { Hemoglobin A1c }(\%) \\
\qquad(\mathrm{mmol} / \mathrm{mol}), \text { IFCC }\end{array}$}} & $6.0 \pm 0.1$ & $5.9 \pm 0.0$ & $6.0 \pm 0.1$ & $6.2 \pm 0.1$ \\
\hline & & $43 \pm 1.6$ & $42 \pm 0.5$ & $42.0 \pm 0.8$ & $43.6 \pm 0.5$ \\
\hline
\end{tabular}

$p$-value vs. Ze 0\% (Dunnett's test). ${ }^{*} p<0.05,{ }^{* *} p<0.01$. The values are presented as mean \pm SE $(n=7)$. 
the 14th week as an indicator of insulin sensitivity demonstrated no significant difference between the Ze $0 \%$ group and any of the zeolite intake groups (Fig. 5).

Plasma lipids. Variations in the levels of plasma lipids are shown in Table 5. In the 8th week, no significant difference was evident in any of the parameters between the Ze $0 \%$ group and any of the zeolite intake groups. On the other hand, in the 12th week, all parameters in the Ze $10 \%$ group were significantly decreased relative

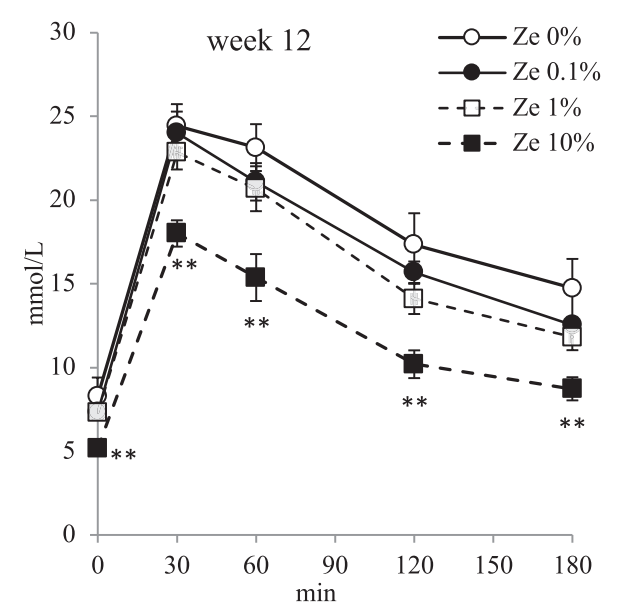

Fig. 3. Changes in blood glucose level in the oral glucose tolerance test at the 12 th week in mice with highfat diet-induced obesity. $p$-value vs. Ze 0\% (Dunnett's test). ${ }^{* *} p<0.01$. The values are presented as mean \pm SE $(n=7)$.

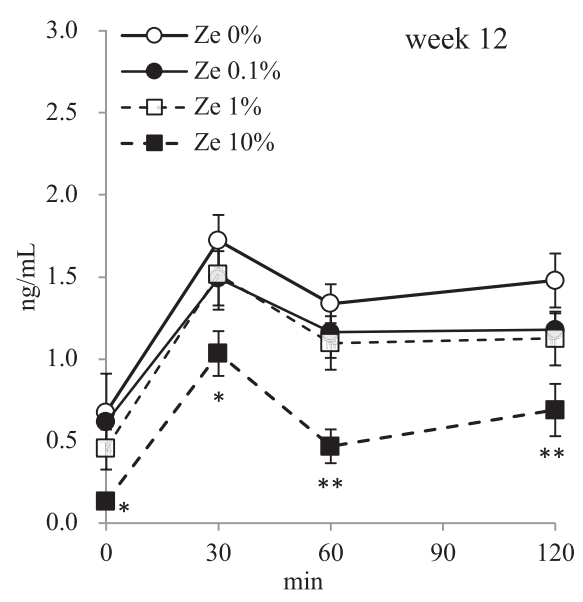

Fig. 4. Changes in plasma insulin levels in the oral glucose tolerance test at the 12th week in mice with highfat diet-induced obesity. $p$-value vs. Ze 0\% (Dunnett's test). ${ }^{*} p<0.05,{ }^{* *} p<0.01$. The values are presented as mean \pm SE $(n=7)$. to the Ze 0\% group. All parameters except for HDL-c were significantly decreased in the Ze $10 \%$ group relative to the Ze $0 \%$ group in the 16 th week. In the 18 th week, only the levels of triacylglycerol and total cholesterol were significantly decreased in the Ze 10\% group relative to the Ze $0 \%$ group.

Other biochemical parameters. The data for other common biochemical parameters are shown in Table 6 . The levels of albumin, amylase, lactate dehydrogenase, potassium and total protein were reduced in parallel with zeolite intake, and the decreases were significant in the Ze $10 \%$ group relative to the Ze $0 \%$ group. On the other hand, the levels of total bilirubin and urea nitrogen increased in parallel with zeolite intake, the increases being significant in the Ze $10 \%$ group relative to the Ze $0 \%$ group. As markers of tissue injury, there were no noteworthy inter-group differences in the activities of alanine aminotransferase and aspartate aminotransferase. There were also no significant inter-group differences in the levels of calcium, inorganic phosphorus, free fatty acids and sodium.

\section{DISCUSSION}

The present study was designed to investigate the influence of single-dose and chronic administration of zeolite on mice with high-fat diet-induced obesity and type 2 diabetes mellitus.

Clinoptilolite, mordenite and non-fibrous Japanese zeolite which are the most common zeolite in nature are considered safe $(12,13)$. On the other hand, fibrous

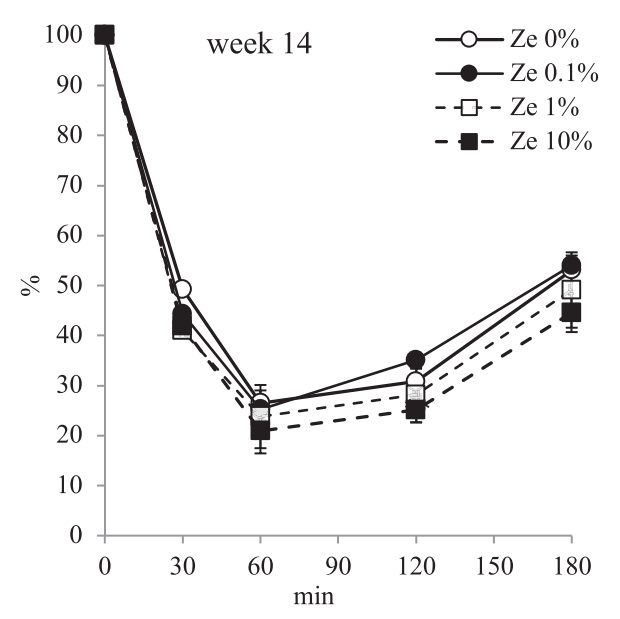

Fig. 5. Changes in blood glucose levels in the insulin tolerance test at the 14th week in mice with high-fat diet-induced obesity. The values are presented as mean \pm SE $(n=7)$.

Table 4. Total area under the curve (tAUC) and incremental area under the curve (iAUC) in mice with diet-induced obesity determined by OGTTs at the 12 th week.

\begin{tabular}{ccccc}
\hline Parameters & Ze 0\% & Ze 0.1\% & Ze 1\% & Ze 10\% \\
\hline tAUC & $60,902 \pm 4,438$ & $55,804 \pm 1,748$ & $52,708 \pm 2,469$ & $39,339 \pm 2,553^{* *}$ \\
iAUC & $34,031 \pm 2,856$ & $32,044 \pm 1,683$ & $29,025 \pm 2,378$ & $22,547 \pm 2,311^{* *}$ \\
\hline
\end{tabular}

$p$-value vs. Ze $0 \%$ (Dunnett's test). ${ }^{* *} p<0.01$. The values are presented as mean $\pm \operatorname{SE}(n=7)$. 
Table 5. Changes in plasma lipid profiles in mice with high-fat diet-induced obesity after zeolite intake.

\begin{tabular}{|c|c|c|c|c|c|}
\hline \multicolumn{2}{|l|}{ Parameters } & \multirow{2}{*}{$\begin{array}{c}\text { Ze } 0 \% \\
0.91 \pm 0.06\end{array}$} & \multirow{2}{*}{$\begin{array}{c}\text { Ze } 0.1 \% \\
0.93 \pm 0.04\end{array}$} & \multirow{2}{*}{$\begin{array}{c}\text { Ze } 1 \% \\
0.85 \pm 0.10\end{array}$} & \multirow{2}{*}{$\begin{array}{r}\text { Ze } 10 \% \\
0.78 \pm 0.05\end{array}$} \\
\hline Triacylglycerol (mmol/L) & week 8 & & & & \\
\hline & week 12 & $1.30 \pm 0.08$ & $1.25 \pm 0.03$ & $1.13 \pm 0.14$ & $0.81 \pm 0.07^{* *}$ \\
\hline & week 16 & $1.08 \pm 0.04$ & $1.02 \pm 0.04$ & $0.92 \pm 0.06^{*}$ & $0.88 \pm 0.05^{*}$ \\
\hline & week 18 & $0.83 \pm 0.05$ & $0.96 \pm 0.07$ & $0.70 \pm 0.03$ & $0.51 \pm 0.06^{* *}$ \\
\hline \multirow[t]{4}{*}{ Total cholesterol $(\mathrm{mmol} / \mathrm{L})$} & week 8 & $3.95 \pm 0.09$ & $4.10 \pm 0.07$ & $3.76 \pm 0.15$ & $3.71 \pm 0.12$ \\
\hline & week 12 & $3.21 \pm 0.13$ & $3.17 \pm 0.05$ & $2.96 \pm 0.13$ & $2.37 \pm 0.07^{* *}$ \\
\hline & week 16 & $3.80 \pm 0.19$ & $3.63 \pm 0.07$ & $3.63 \pm 0.11$ & $3.19 \pm 0.15^{* *}$ \\
\hline & week 18 & $2.84 \pm 0.20$ & $2.89 \pm 0.03$ & $2.57 \pm 0.11$ & $2.44 \pm 0.05^{*}$ \\
\hline \multirow[t]{4}{*}{ HDL-c (mmol/L) } & week 8 & $2.70 \pm 0.04$ & $2.75 \pm 0.03$ & $2.56 \pm 0.06$ & $2.53 \pm 0.07$ \\
\hline & week 12 & $2.08 \pm 0.07$ & $2.14 \pm 0.03$ & $2.06 \pm 0.05$ & $1.80 \pm 0.04^{* *}$ \\
\hline & week 16 & $2.30 \pm 0.08$ & $2.25 \pm 0.04$ & $2.30 \pm 0.06$ & $2.15 \pm 0.08$ \\
\hline & week 18 & $1.47 \pm 0.04$ & $1.53 \pm 0.02$ & $1.39 \pm 0.04$ & $1.35 \pm 0.05$ \\
\hline \multirow[t]{4}{*}{ Non-HDL-c (mmol/L) } & week 8 & $1.25 \pm 0.09$ & $1.34 \pm 0.05$ & $1.20 \pm 0.10$ & $1.18 \pm 0.06$ \\
\hline & week 12 & $1.14 \pm 0.13$ & $1.04 \pm 0.07$ & $0.90 \pm 0.09$ & $0.58 \pm 0.06^{* *}$ \\
\hline & week 16 & $1.50 \pm 0.16$ & $1.37 \pm 0.07$ & $1.33 \pm 0.09$ & $1.04 \pm 0.07^{* *}$ \\
\hline & week 18 & $1.37 \pm 0.17$ & $1.36 \pm 0.03$ & $1.18 \pm 0.09$ & $1.09 \pm 0.02$ \\
\hline \multirow[t]{4}{*}{ Non-HDL-c/HDL-c ratio } & week 8 & $0.47 \pm 0.03$ & $0.49 \pm 0.02$ & $0.47 \pm 0.03$ & $0.46 \pm 0.02$ \\
\hline & week 12 & $0.55 \pm 0.08$ & $0.49 \pm 0.04$ & $0.44 \pm 0.04$ & $0.32 \pm 0.03^{* *}$ \\
\hline & week 16 & $0.65 \pm 0.07$ & $0.61 \pm 0.03$ & $0.58 \pm 0.04$ & $0.48 \pm 0.02^{* *}$ \\
\hline & week 18 & $0.93 \pm 0.09$ & $0.89 \pm 0.03$ & $0.85 \pm 0.06$ & $0.82 \pm 0.04$ \\
\hline
\end{tabular}

$p$-value vs. Ze 0\% (Dunnett's test). ${ }^{*} p<0.05,{ }^{* *} p<0.01$. The values are presented as mean \pm SE $(n=7)$.

Table 6. Plasma biochemical parameters in mice with high-fat diet-induced obesity after zeolite intake for $18 \mathrm{wk}$.

\begin{tabular}{|c|c|c|c|c|}
\hline Parameters & Ze $0 \%$ & Ze $0.1 \%$ & Ze $1 \%$ & Ze $10 \%$ \\
\hline Alanine aminotransferase ( $\mu$ kat/L) & $1.18 \pm 0.50$ & $1.01 \pm 0.29$ & $0.59 \pm 0.11$ & $0.46 \pm 0.03$ \\
\hline Albumin $(\mathrm{g} / \mathrm{L})$ & $32 \pm 0.8$ & $32 \pm 0.6$ & $31 \pm 0.9$ & $28 \pm 0.9^{* *}$ \\
\hline Amylase $(\mu \mathrm{kat} / \mathrm{L})$ & $57.1 \pm 1.80$ & $62.2 \pm 1.55$ & $53.3 \pm 2.82$ & $47.9 \pm 2.16^{* *}$ \\
\hline Aspartate aminotransferase ( $\mu \mathrm{kat} / \mathrm{L})$ & $2.94 \pm 0.51$ & $3.04 \pm 0.45$ & $2.85 \pm 0.57$ & $2.21 \pm 0.60$ \\
\hline Calcium $(\mathrm{mmol} / \mathrm{L})$ & $2.18 \pm 0.03$ & $2.28 \pm 0.03^{*}$ & $2.22 \pm 0.01$ & $2.25 \pm 0.01$ \\
\hline Chloride (mmol/L) & $110 \pm 2.0$ & $113 \pm 0.4^{*}$ & $114 \pm 0.9^{*}$ & $114 \pm 0.5^{*}$ \\
\hline Creatinine $(\mu \mathrm{mol} / \mathrm{L})$ & $15 \pm 1.3$ & $13 \pm 0.6$ & $13 \pm 0.7$ & $14 \pm 0.7$ \\
\hline Inorganic phosphorus (mmol/L) & $2.67 \pm 0.18$ & $2.77 \pm 0.12$ & $2.69 \pm 0.06$ & $2.72 \pm 0.12$ \\
\hline Lactate dehydrogenase ( $\mu$ kat/L) & $9.61 \pm 2.19$ & $7.14 \pm 0.75$ & $7.42 \pm 1.01$ & $5.59 \pm 0.62 *$ \\
\hline Non-esterified fatty acids (mmol/L) & $0.65 \pm 0.04$ & $0.60 \pm 0.03$ & $0.64 \pm 0.05$ & $0.59 \pm 0.04$ \\
\hline Potassium $(\mathrm{mmol} / \mathrm{L})$ & $10.0 \pm 0.6$ & $9.6 \pm 0.4$ & $7.9 \pm 0.3^{* *}$ & $7.3 \pm 0.2^{* *}$ \\
\hline Sodium $(\mathrm{mmol} / \mathrm{L})$ & $150 \pm 1.4$ & $150 \pm 0.4$ & $153 \pm 1.3$ & $152 \pm 0.2$ \\
\hline Total bilirubin $(\mu \mathrm{mol} / \mathrm{L})$ & $0.4 \pm 0.1$ & $0.4 \pm 0.1$ & $0.6 \pm 0.1$ & $0.7 \pm 0.1^{* *}$ \\
\hline Total protein $(\mathrm{g} / \mathrm{L})$ & $52 \pm 1.4$ & $52 \pm 0.7$ & $51 \pm 1.2$ & $48 \pm 1.1^{*}$ \\
\hline Urea nitrogen $(\mathrm{mmol} / \mathrm{L})$ & $8.3 \pm 0.4$ & $8.3 \pm 0.2$ & $9.1 \pm 0.3$ & $9.4 \pm 0.4^{*}$ \\
\hline
\end{tabular}

$p$-value vs. Ze $0 \%$ (Dunnett's test). ${ }^{*} p<0.05,{ }^{* *} p<0.01$. The values are presented as mean \pm SE $(n=7)$.

mordenite can be considered a potential health hazard (16), but there is no evidence to support this. The contents of fibrous mordenite in the zeolite used in this study was not measured. However, over a 14-d monitoring period after single-dose administration of zeolite $(2,000 \mathrm{mg} / \mathrm{kg} \mathrm{bw})$, no abnormality or death was observed. Furthermore, necropsy also revealed no abnormality in the mice. Therefore, in both male and female mice, the lowest lethal dose (LDLo) of the zeolite preparation used in this study was speculated to be more than $2,000 \mathrm{mg} / \mathrm{kg}$ bw. Other oral toxicity studies have also supported the high safety of zeolites $(17,18)$.

Intake of the zeolite at the maximum dose suppressed the increases of both body weight and visceral fat in the model animals (Fig. 2, Table 2). Therefore, from the viewpoint of weight control, the recommended daily 
intake of dietary zeolite in this study would range from $1 \%$ to $10 \%$. Because there were no significant intergroup differences in food or energy intake (Fig. 1, Table 2 ), the contribution of either parameter to the prevention of both body weight and visceral fat gain was denied. Although neither kidney nor pancreas weight changed, liver weight decreased in parallel with zeolite intake. Although liver lipid content was not measured, the decreases in the levels of plasma lipids (triacylglycerol, total cholesterol and HDL-c) in accordance with zeolite intake (Table 5) suggested that the decrease in liver weight was due to inhibition of liver lipid accumulation. Because natural zeolite is not absorbed through the gastrointestinal tract (1), its in vivo effects cannot be due to direct biochemical interaction. Zeolite has a micropore structure framework consisting of silicon dioxide. Although the pore structure is unknown, it would be impossible for macromolecules such as triacylglycerol, free fatty acid, cholesterol and glucose to enter the pores because the pore diameter of common zeolite is approximately $1 \mathrm{~nm}$. However, zeolite inhibits gastrointestinal reabsorption of thyroid hormone (19), bile acids (20), and gastrointestinal absorption of cholesterol (19). Furthermore, zeolite is a strong sorbent for fructose, glucose, sucrose, fructooligosaccharides, lipids, and proteins (5-8). All of the above reports support the present findings that zeolite intake decreased the level of blood glucose and the plasma levels of lipid and albumin. Zeolite can reduce the level of blood glucose through its well known adsorption properties (4), and therefore its use as additional therapy for diabetes mellitus is expected $(21,22)$. The present study suggested that gastrointestinal absorption of macronutrients might be inhibited through multiple interactions resulting from the adsorptive properties of zeolite, as reflected in the observed inhibition of body weight gain and visceral fat accumulation (Table 2).

Obesity and visceral fat accumulation are risk factors for type 2 diabetes mellitus and are frequently associated with hyperlipidemia. Because type 2 diabetes mellitus and hyperlipidemia due to obesity were predominant in this study, the levels of fasting blood glucose (Table 3) and plasma lipids (Table 5) would have been suppressed synchronously due to the inhibition of body weight gain by zeolite intake. The observed decrease in the fasting blood glucose level (Table 3) represents one piece of evidence that zeolite had an ameliorating effect on high-fat diet-induced diabetes. However, during the study period, inhibition of the fasting blood glucose level by zeolite intake was not a constant feature (Table 3). HbA1c shows a stronger relationship with the fasting blood glucose level than with the postprandial blood glucose level $(23,24)$. This may explain why zeolite had no apparent effect on $\mathrm{HbAlc}$, which is a persistent blood glucose parameter (Table 3). The Collaborative Analysis of Diagnostic Criteria in Europe (DECODE) study demonstrated that cardiovascular mortality was correlated with the peak glucose level within $2 \mathrm{~h}$ of glucose loading, suggesting that the risk of death among diabetic patients cannot be discriminated purely on the basis of fasting blood glucose data (25). The suppressive effect of zeolite on the postprandial increase in blood glucose was more obvious with increased zeolite intake, and intake of the maximal dose elicited a significant effect (Fig. 3, Table 4). Even though the chronicity study revealed no clear effect of zeolite on $\mathrm{HbA} 1 \mathrm{c}$, because suppression of postprandial hyperglycemia is important for prevention of diabetes and cardiovascular mortality, the observed suppressive effect was considered at least potentially promising for diabetes prevention. Although the ITT at the 14 th week demonstrated no significant difference (Fig. 5), it was suggested that decreased insulin secretion (Fig. 4) accompanying postprandial blood glucose suppression (Fig. 3) may have resulted from improvement of insulin sensitivity through suppression of body weight gain (Table 2).

With regard to mineral levels, maximal intake of the zeolite preparation reduced the level of potassium by $26 \%$ without any change in the level of either calcium or phosphorus (Table 6). In contrast, Martin-Kleiner et al. (26) detected a 20\% increase in the level of potassium without any other changes in serum chemistry in mice receiving a zeolite-rich diet. The differences in these findings might be due to differences in the adsorption properties of natural zeolite, which are affected by the $\mathrm{Al} / \mathrm{Si}$ ratio, the micropore structure framework, and the metal ion replaced in the framework. Martin-Kleiner et al. (26) showed that basic kidney (creatinine) and liver (bilirubin) function parameters in mice ingesting clinoptilolite-rich tuff remained within reference values. In the present study, the level of urea nitrogen increased in parallel with zeolite intake, but the creatinine level remained normal (Table 6). Therefore, it is unlikely that the experimental animals developed any significant kidney dysfunction. Because the $10 \%$ zeolite (highest content) pellets were relatively fragile, they often dropped from the food container on to the floor of the cage. One tentative explanation is that the food was contaminated with urine, because mice consumed food crumbs from the bedding material. The observed decrease of lactate dehydrogenase activity suggested suppression and/or improvement of some form of tissue injury (Table 6). Moreover, the decrease of amylase activity in accordance with zeolite intake suggested suppression and/or improvement of pancreatic function (Table 6). However, a recent study (27) has shown that low serum levels of amylase are significantly associated with type 2 diabetes mellitus, excess adiposity, and metabolic syndrome. On the other hand, the increase in the bilirubin level with continued zeolite intake appeared to suggest the development of liver dysfunction. However, in a clinical study, Zheng et al. (28) have reported that a lower serum total bilirubin level was associated with enhancement of the inflammatory response in patients with impaired glucose tolerance and type 2 diabetes mellitus. Therefore, the chronic toxicity of zeolite will need to be investigated further.

Our study had several limitations. We did not determine the contents of fibrous mordenite in the natural zeolite used in this study. In addition, we did not identify 
the micropore framework structure of the natural zeolite and the species of metal ion replaced in the framework. Therefore, the electric charge balance was unknown; however, potential ability to preferentially adsorb potassium ions was found. The adsorption properties of the natural zeolite may be associated with the action mechanism, but they remain a matter of inference.

In conclusion, the present study has determined the LDLo of this new natural zeolite preparation and shown that when taken as a dietary component it is able to improve hyperglycemia, insulin sensitivity and chronic lipid metabolism disorders in model mice with high-fat diet-induced obesity and type 2 diabetes mellitus.

\section{Authorship}

Research conception, design, and experiments: KK and YK; procurement and refining of zeolite: YK; statistical analysis of the data, interpretation of the data and writing of the manuscript: KK.

\section{Disclosure of state of COI}

KK has no competing financial or non-financial interests, has not received any consulting fees/honoraria, has no leadership/advisory role in the company, and receives no patent royalties/licensing fees or other benefits (e.g., gifts). Because YK is an employee of Bikensangyo Co., Ltd., he has a financial competing interest, a non-financial competing interest, has received a salary, and has a leadership/advisory role in the company.

\section{Acknowledgments}

The authors gratefully acknowledge the technical assistance of Ms. Rina Konishi and Ms. Ayaka Tsuji.

\section{Funding}

This study was performed as part of a Joint Research Agreement between Gifu University and Bikensangyo Co., Ltd. KK received research funding through Gifu University, which is supported by Bikensangyo Co., Ltd.

\section{Supporting information}

Supplemental online material is available on J-STAGE.

\section{REFERENCES}

1) Pavelic K, Katic M, Sverko V, Marotti T, Bosnjak B, Balog T, Stojkovic R, Radacic M, Colic M, Poljak-Blazi M. 2002. Immunostimulatory effect of natural clinoptilolite as a possible mechanism of its antimetastatic ability. J Cancer Res Clin Oncol 128: 37-44.

2) Nagy JB, Bodart P, Hanus I, Kiricsi I (Eds). 1998. Ion exchange, sorption and diffusion in microporous materials. In: Synthesis, Characterization and Use of Zeolitic Microporous Materials, p 93-118. DecaGen Ltd, Szeged.

3) Mumpton FA. 1999. La roca magica: uses of natural zeolites in agriculture and industry. Proc Natl Acad Sci USA 96: 3463-3470.

4) Cisneros V, Ocanto F, Linares CF. 2011. $\mathrm{Ca}^{2+}, \mathrm{Mg}^{2+}$ OR $\mathrm{Fe}^{2+}$ ion-exchanged cancrinitetype zeolites as possible hypoglycemiant agents. Rev Latinoam Quim 39: 55-61.

5) Kuhn RC, Mazutti MA, Maugeri Filho F. 2012. Kinetic and mass transfer effects for adsorption of glucose, fructose, sucrose and fructooligosaccharides into $\mathrm{X}$ zeolite. LWT-Food Sci Technol 48: 127-133.

6) Bai P, Siepmann JI, Deem MW. 2013. Adsorption of glucose into zeolite beta from aqueous solution. AIChE J 59: 3523-3529.

7) Francisco M, Mlinar AN, Yoo B, Bell AT, Prausnitz JM. 2011. Recovery of glucose from an aqueous ionic liquid by adsorption onto a zeolite-based solid. Chem Eng J 172: 184-190.

8) Pavelić K, Hadzija M, Bedrica L, Pavelić J, Dikić I, Katić M, Kralj M, Bosnar MH, Kapitanović S, Poljak-Blazi M, Krizanac S, Stojković R, Jurin M, Subotić B, Colić M. 2001. Natural zeolite clinoptilolite: new adjuvant in anticancer therapy. J Mol Med (Berl) 78: 708-720.

9) Kubo K, Koido A, Kitano M, Yamamoto H, Saito M. 2016. Combined effects of a dietary fiber mixture and wheat albumin in a rat model of type 2 diabetes mellitus. J Nutr Sci Vitaminol 62: 416-424.

10) Oschiliewski U, Kiesel U, Kolb H. 1985. Administration of silica prevents diabetes in BB-rats. Diabetes Res Inst 34: 197-199.

11) Kraljević Pavelić S, Simović Medica J, Gumbarević D, Filošević A, Pržulj N, Pavelić K. 2018. Critical review on zeolite clinoptilolite safety and medical applications in vivo. Front Pharmacol 27: 1350.

12) EFSA Panel on Additives and Products or Substances used in Animal Feed. 2013. Scientific opinion on the safety and efficacy of clinoptilolite of sedimentary origin for all animal species. EFSA J 11: 1-14.

13) EFSA Panel on Additives and Products or Substances used in Animal Feed. 2013. Scientific opinion on the safety and efficacy of clinoptilolite of sedimentary origin for all animal species. EFSA J 11: 3039.

14) Organization of Economic Co-operation and Development. 2001. OECD guideline for testing of chemicals: Guideline 420. Acute Oral Toxicity-Fixed Dose Procedure. OECD, Paris, France.

15) Organization of Economic Co-operation and Development. 2018. OECD guideline for testing of chemicals: Guideline 408. Repeated Dose 90-Day Oral Toxicity Study in Rodents. OECD, Paris, France.

16) Wright WE, Rom WN, Moatamed F. 1983. Characterization of zeolite fiber sizes using scanning electron microscopy. Arch Environ Health 38: 99-103.

17) Elmore AR. 2003. Cosmetic Ingredient Review Expert Panel. Final report on the safety assessment of aluminum silicate, calcium silicate, magnesium aluminum silicate, magnesium silicate, magnesium trisilicate, sodium magnesium silicate, zirconium silicate, attapulgite, bentonite, Fuller's earth, hectorite, kaolin, lithium magnesium silicate, lithium magnesium sodium silicate, montmorillonite, pyrophyllite, and zeolite. Int J Toxicol 22 (Suppl 1): 37-102.

18) Krajišnik D, Stepanović-Petrović R, Tomić M, Micov A, Ibrić S, Milić J. 2014. Application of artificial neural networks in prediction of diclofenac sodium release from drug-modified zeolites physical mixtures and antiedematous activity assessment. J Pharm Sci 103: 10851094.

19) Cai Y, Meng XF, Cao YX, Lu H, Zhu SF, Zhou LZ. 2006. Montmorillonite ameliorates hyperthyroidism of rats and mice attributed to its adsorptive effect. Eur J Pharmacol 551: 156-161.

20) Cutovic M, Lazovic M, Vukovic-Dejanovic V, Nikolic D, 
Petronic-Markovic I, Cirovic D. 2017. Clinoptilolite for treatment of dyslipidemia: Preliminary efficacy study. $J$ Altern Complement Med 23: 738-744.

21) Concepción-Rosabal B, Rodriguez-Fuentes G, SimónCarballo R. 1997. Development and featuring of the zeolitic active principle FZ: A glucose adsorbent. Zeolites 19: $47-50$.

22) Zarkovic N, Zarkovic K, Kralj M, Borovic S, Sabolovic S, Blazi MP, Cipak A, Pavelic K. 2003. Anticancer and antioxidative effects of micronized zeolite clinoptilolite. Anticancer Res 23: 1589-1595.

23) Borg R, Kuenen JC, Carstensen B, Zheng H, Nathan DM, Heine RJ, Nerup J, Borch-Johnsen K, Witte DR; ADAG Study Group. 2010. Associations between features of glucose exposure and A1C: the A1C-Derived Average Glucose (ADAG) study. Diabetes 59: 1585-1590.

24) Bonora E, Calcaterra F, Lombardi S, Bonfante N, Formentini G, Bonadonna RC, Muggeo M. 2001. Plasma glucose levels throughout the day and $\mathrm{HbA}(1 \mathrm{c})$ interrelationships in type 2 diabetes: implications for treatment and monitoring of metabolic control. Diabetes Care 24: 2023-2029.
25) The DECODE Study Group. European Diabetes Epidemiology Group. Diabetes Epidemiology: Collaborative analysis Of Diagnostic criteria in Europe. 1999. Glucose tolerance and mortality: comparison of WHO and American Diabetes Association diagnostic criteria. Lancet 354: 617-621.

26) Martin-Kleiner I, Flegar-Mestric Z, Zadro R, Breljak D, Stanovic Janda S, Stojkovic R, Marusic M, Radacic M, Boranic M. 2001. The effect of the zeolite clinoptilolite on serum chemistry and hematopoiesis in mice. Food Chem Toxicol 39: 717-727.

27) Ko J, Cho J, Petrov MS. 2020. Low serum amylase, lipase, and trypsin as biomarkers of metabolic disorders: A systematic review and meta-analysis. Diabetes Res Clin Pract 159: 107974.

28) Zheng J, Wu Y, Li Z, Wang H, Xiao W, Shi Y, Wang T. 2016. Low serum total bilirubin concentration was associated with increased high sensitive $\mathrm{C}$ reactive protein level in patients with impaired glucose tolerance and type 2 diabetes mellitus subjects. Clin Lab 62: 901907. 